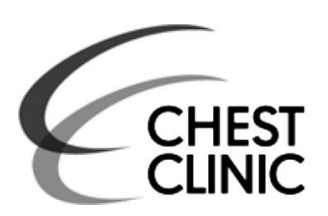

AUDIT, RESEARCH AND GUIDELINE UPDATE

\title{
Role of CT in assessing pleural malignancy prior to thoracoscopy
}

\author{
R J Hallifax, ${ }^{1}$ M Haris, ${ }^{2}$ J P Corcoran, ${ }^{1}$ S Leyakathalikhan, ${ }^{2}$ E Brown, ${ }^{3}$ \\ D Srikantharaja, ${ }^{3}$ A Manuel, ${ }^{1}$ F V Gleeson, ${ }^{4}$ M Munavvar, $^{2}$ N M Rahman ${ }^{1}$
}

${ }^{1}$ Oxford Centre for Respiratory Medicine, Oxford University Hospitals NHS Trust, Oxford, UK

${ }^{2}$ Lancashire Chest Centre, Lancashire Teaching Hospitals, Preston, UK

${ }^{3}$ University of Oxford, Clinical Medical School, Oxford, UK ${ }^{4}$ Department of Radiology, Oxford University Hospitals NHS Trust, Oxford, UK

\section{Correspondence to} Dr Rob Hallifax, Oxford Respiratory Trials Unit, University of Oxford, Churchill Hospital, Oxford, OX3 7LJ, UK robhallifax@yahoo.com

Received 14 July 2014 Accepted 17 July 2014 Published Online First 30 July 2014

\section{CrossMark}

To cite: Hallifax RJ, Haris M, Corcoran JP, et al. Thorax 2015;70:192-193.

\section{ABSTRACT}

The definitive diagnosis of pleural malignancy depends upon histological confirmation by pleural biopsy. CT is reported to have a high sensitivity and specificity for the diagnosis of malignant pleural disease, and is part of the routine diagnostic workup of these patients. The aim of this study was to assess the sensitivity and specificity of $\mathrm{CT}$ in detecting pleural malignancy prior to definitive histology obtained via thoracoscopy in a large cohort of patients with suspected malignant pleural disease. Retrospective review of thoracoscopies between January 2008 and January 2013 at two UK tertiary referral centres: Oxford and Preston. The histological results were compared with the $\mathrm{CT}$ reported diagnosis before the procedure. CT scan reports were assessed by independent respiratory physicians as to whether the radiologist concluded evidence of malignant pleural disease or benign features only. 211 (57\%) of 370 patients included in the analysis had malignant disease: CT scans were reported as 'malignant' in 144, giving a sensitivity of $68 \%(95 \% \mathrm{Cl} 62 \%$ to $75 \%)$. Of the 159 patients with benign disease, 124 had CT scans reported as benign: specificity $78 \%$ ( $72 \%$ to $84 \%)$. The positive predictive value of a malignant CT report was $80 \%(75 \%$ to $86 \%)$, with a negative predictive value of $65 \%$ (58\% to $72 \%$ ). A significant proportion of patients being investigated for malignant disease will have malignancy despite a negative $\mathrm{CT}$ report. The use of $\mathrm{CT}$ alone in determining which patients should have invasive pleural biopsies should be re-evaluated, and further studies to define the diagnostic pathway are now required.

\section{BACKGROUND}

Pleural disease is common, affecting over 300 people per 100000 of the population each year. ${ }^{1}$ The definitive diagnosis of pleural malignancy depends upon histological confirmation by pleural biopsy. Contrast-enhanced thoracic CT is reported to have a high sensitivity and specificity to help differentiate between benign and malignant disease, and is therefore an essential part of the diagnostic workup of these patients. Features of malignant disease on CT scanning are nodular pleural thickening, mediastinal pleural thickening, parietal pleural thickening $>1 \mathrm{~cm}$ and circumferential pleural thickening. One small study found that these features had specificities of $94 \%, 94 \%, 88 \%$ and $100 \%$, respectively, and sensitivities of $51 \%, 36 \%, 56 \%$ and $41 \%{ }^{2}$ Subsequent studies assessing these criteria for the detection of pleural malignancy reported high sensitivities and specificities. ${ }^{3}{ }^{4}$ However, studies were carried out in relatively small cohorts of $40^{3}$ and 45 patients $^{4}$ over a decade ago, and there is, therefore, the need for further data appropriate to current practice.

The aim of this study was to assess the sensitivity and specificity of CT in detecting pleural malignancy (primary and metastatic) prior to definitive histology obtained via thoracoscopy in a large cohort of patients with suspected malignant pleural disease.

\section{METHODS}

Retrospective review of thoracoscopy procedures were carried out between January 2008 and January 2013 at two UK tertiary referral centres: Churchill Hospital, Oxford and Lancashire Teaching Hospitals, Preston. Four hundred and fifty thoracoscopies were performed to investigate new pleural effusions over the 5 -year period. Forty-seven patients did not have CT scans prior to having thoracoscopy or their formal CT report was not available (eg, if the images had been imported from an external source), so were not included. In 33 instances, cases were excluded as either the biopsy was non-diagnostic or no biopsy was performed (ie, thoracoscopy was performed for talc pleurodesis only). Therefore, 370 cases were included in the analysis. One hundred and ninety patients were investigated for a new pleural effusion with evidence of pleural thickening; 136 had unexplained effusion (without evidence of thickening on prior imaging), 40 had new effusion in the context of current or previous extrapulmonary malignancy and 4 had an effusion with suspected primary lung carcinoma.

The histological results from thoracoscopy were compared with the CT reported diagnosis before the procedure. The CT scan reports were assessed by a number of independent respiratory trainee physicians as to whether the reporting radiologist provided conclusive evidence of malignant pleural disease in the written report, or reported benign features only. Each case was reassessed by a senior respiratory trainee; those in which there was discrepancy were re-examined by two senior assessors for agreement. Reports which were 'highly suggestive or suggestive' of malignant disease were classified as malignant; those which concluded 'benign features', 'not conclusive' or 'raising the possibility of malignancy' were classified as benign. Patients with benign histology (ie, negative for pleural malignancy) remained under clinical surveillance for at least 2 years, reflecting the known $\sim 8 \%$ of cases which are proven to be malignant over follow-up in cases of 'non-specific pleuritis'. 
Table 1 Sensitivity and specificity of CT scan reporting malignancy by indication for thoracoscopic investigation

\begin{tabular}{|c|c|c|c|c|c|}
\hline Indication & Sensitivity $(95 \% \mathrm{Cl})$ & Specificity $(95 \% \mathrm{Cl})$ & PPV $(95 \% \mathrm{Cl})$ & NPV $(95 \% \mathrm{Cl})$ & Total no. \\
\hline Unexplained effusion & $69 \%(62 \%$ to $76 \%)$ & $78 \%(72 \%$ to $85 \%)$ & $80 \%(74 \%$ to $87 \%)$ & $66 \%(59 \%$ to $74 \%)$ & 326 \\
\hline Effusion in known/previous malignancy & $64 \%(47 \%$ to $82 \%)$ & $75 \%(54 \%$ to $96 \%)$ & $82 \%(66 \%$ to $98 \%)$ & $55 \%(34 \%$ to $75 \%)$ & 44 \\
\hline Overall & $68 \%(62 \%$ to $75 \%)$ & $78 \%(72 \%$ to $84 \%)$ & $80 \%$ ( $75 \%$ to $86 \%)$ & $65 \%$ (58\% to $72 \%)$ & 370 \\
\hline
\end{tabular}

NPV, negative predictive value; PPV, positive predictive value.

Sensitivity and specificity were calculated using the final histological diagnosis as the gold standard by which the CT diagnostic accuracy is tested. CIs were calculated using the Wald method as the sample size was sufficient (370 patients) and the values were not at the extreme ends of probability (ie, neither near 0 nor 1 ).

\section{RESULTS}

Of the 370 patients included in the analysis, 261 (70.5\%) were men. The mean age was 72.3 years (SD 12.9 years). On histological analysis of the biopsies taken at thoracoscopy, 202 $(54.6 \%)$ of the 370 patients had malignant pleural disease: 110 $(54.5 \%)$ of 202 were mesothelioma; $92(45.5 \%)$ were metastatic carcinoma from another primary carcinoma. The remaining 167 (45.4\%) of 370 had benign disease: 149 (88.7\%) of 167 showed benign chronic inflammation and fibrosis, $10(6.0 \%)$ had pleural TB, 4 (2.4\%) had granulomatous disease of other aetiology, $3(1.8 \%)$ had evidence of mesothelial hyperplasia, 1 $(0.6 \%)$ had fibrosis in keeping with rheumatoid disease and 1 $(0.6 \%)$ was diagnosed with malakoplakia (a rare inflammatory condition causing a papule, plaque or ulceration; which usually affects the genitourinary tract). Routine practice is that all patients with chronic inflammation and fibrosis on histology are followed up for 2 years as surveillance for evidence of clinical or radiological development of pleural malignancy. During this period, $9(6.0 \%)$ of the 149 patients were subsequently diagnosed with malignancy: 8 were mesothelioma (6 proven on subsequent biopsy; 2 with clinical and radiological progression only) and 1 metastatic adenocarcinoma from lung primary adenocarcinoma. These nine patients were classified as 'malignant' for the sensitivity and specificity analysis: therefore, 211 of $370(57.0 \%)$ were malignant and 140 (37.8\%) had chronic inflammation only.

\section{Sensitivity and specificity}

Of the 211 patients with malignant disease, 144 had CT scans prior to thoracoscopy reported as malignant (sensitivity $68.2 \%$, $95 \%$ CI $62.0 \%$ to $74.5 \%$ ). Of the 159 patients with benign disease, 124 had CT scans reported as benign (specificity $78.0 \%, 95 \%$ CI $71.5 \%$ to $84.4 \%$ ). The positive predictive value of a 'malignant' CT report was $80.4 \%(74.6 \%$ to $86.3 \%)$, with a negative predictive value of $64.9 \%$ (58.2\% to $71.7 \%)$.

Of the total cohort, $326(88.1 \%)$ patients originally presented with a new pleural effusion of unexplained aetiology. The sensitivity and specificity in these cases were 69\% (95\% CI\% 62 to $76 \%$ ) and $78 \%(72 \%$ to $85 \%)$, respectively. Forty-four (11.9\%) patients presented with an effusion in the context of known or previous malignancy, the sensitivity and specificity were $64 \%$ (95\% CI $47 \%$ to $82 \%$ ) and $75 \%$ (55\% to $96 \%)$, respectively (see table 1$)$.

\section{DISCUSSION}

The use of CT scanning is firmly established in the diagnostic pathway for investigating pleural effusion. Current guidelines suggest that if analysis of pleural fluid ( $\mathrm{pH}$, total protein, lactate dehydrogenase, microbiological culture and cytology) does not reveal a cause, then all undiagnosed exudative effusions should be further investigated with a contrast enhanced CT scan. ${ }^{1}$ The radiological report of this scan will influence how, or indeed whether, a patient is further investigated.

This retrospective study is the largest to date assessing the sensitivity and specificity of CT reporting in patients being investigated for suspected pleural malignancy, and especially in the modern age of comprehensive investigation to evaluate pleural effusion based on current guidelines. ${ }^{1}$ Previous smaller studies have suggested that certain features on CT scan are characteristic of malignant disease (nodular pleural thickening, mediastinal pleural thickening, parietal pleural thickening $>1 \mathrm{~cm}$ and circumferential pleural thickening), with modest sensitivity (36\%$51 \%$ ) but good specificity $(88 \%-100 \%)$. Data presented in this study suggest that although the sensitivity of a malignant CT report is higher than previously reported $(68 \%)$, the specificity was significantly lower (78\%). This difference may reflect the changing patterns of disease or changes in the use of invasive biopsy techniques.

With a negative predictive value of $65 \%$, approximately one in every three patients with pleural effusion and a CT scan reported no evidence of malignant disease of the pleura will in fact have malignancy. Physicians and radiologists should bear this in mind when discussing cases in clinical practice and at multidisciplinary team meetings. Despite the increasing use of CT scanning, these data suggest that the use of CT alone in determining which patients should have invasive pleural biopsies should be re-evaluated, and further studies to define the diagnostic pathway are now required.

Contributors RJH, MH, FVG, MM and NMR were involved in project conception, data analysis, drafting and final approval of the manuscript. JPC, EB, DS and AM performed the majority of data collection and initial data analysis in Oxford; in Preston this was completed by SL and MH. RJH, MH and NMR are the guarantors.

\section{Competing interests None.}

Ethics approval No patient information or patient identifiable images are included in this manuscript. This study was a retrospective review of case notes and therefore did not require submission to ethical approval committee.

Provenance and peer review Not commissioned; internally peer reviewed.

Data sharing statement All data is stored by the corresponding author. There is no additional unpublished data.

\section{REFERENCES}

1 Du Rand I, Maskell N. Introduction and methods: British Thoracic Society pleural disease guideline. Thorax 2010;65(Suppl 2):1-3.

2 Leung AN, Müller NL, Miller RR. CT in differential diagnosis of diffuse pleural disease. Am J Roentgenol 1990;154:487-92.

3 Traill ZC, Davies RJ, Gleeson FV. Thoracic computed tomography in patients with suspected malignant pleural effusions. Clin Radiol 2001;56:193-6.

4 Scott EM, Marshall TJ, Flower CD, et al. Diffuse pleural thickening: percutaneous CT-guided cutting needle biopsy. Radiology 1995;194:867-70.

5 Davies HE, Nicholson JE, Rahman NM, et al. Outcome of patients with nonspecific pleuritis/fibrosis on thoracoscopic pleural biopsies. Eur J Cardiothorac Surg 2010;38:472-7. 